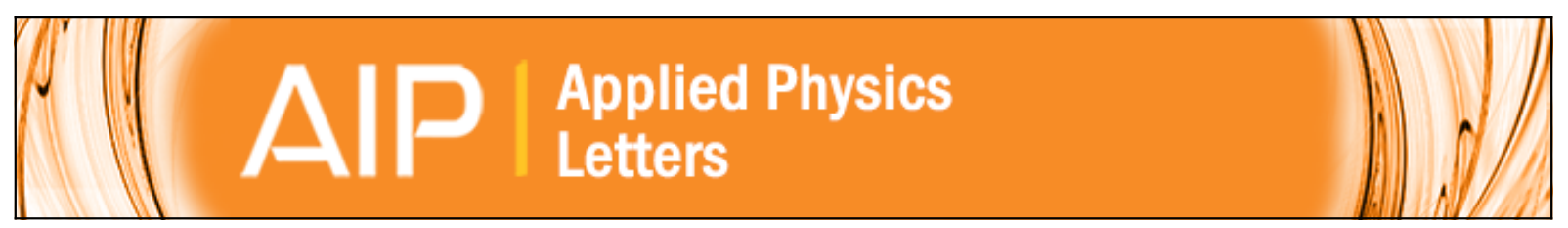

\title{
Photon management with nanostructures on concentrator solar cells
}

J. Buencuerpo, J. M. Llorens, M. L. Dotor, and J. M. Ripalda

Citation: Applied Physics Letters 103, 083901 (2013); doi: 10.1063/1.4819100

View online: http://dx.doi.org/10.1063/1.4819100

View Table of Contents: http://scitation.aip.org/content/aip/journal/apl/103/8?ver=pdfcov

Published by the AIP Publishing

\section{Articles you may be interested in}

Modelling of $\mathrm{GaAsP} / \mathrm{InGaAs} / \mathrm{GaAs}$ strain-balanced multiple-quantum well solar cells

J. Appl. Phys. 113, 024512 (2013); 10.1063/1.4775404

Theoretical analysis and modeling of light trapping in high efficicency GaAs nanowire array solar cells

Appl. Phys. Lett. 99, 143116 (2011); 10.1063/1.3647847

Reducing carrier escape in the InAs/GaAs quantum dot intermediate band solar cell

J. Appl. Phys. 108, 064513 (2010); 10.1063/1.3468520

Low-acceptor-concentration GalnNAs grown by molecular-beam epitaxy for high-current $\mathrm{p}$ - $\mathrm{i}$ - $\mathrm{n}$ solar cell applications

J. Appl. Phys. 98, 094501 (2005); 10.1063/1.2113414

APL Photonics

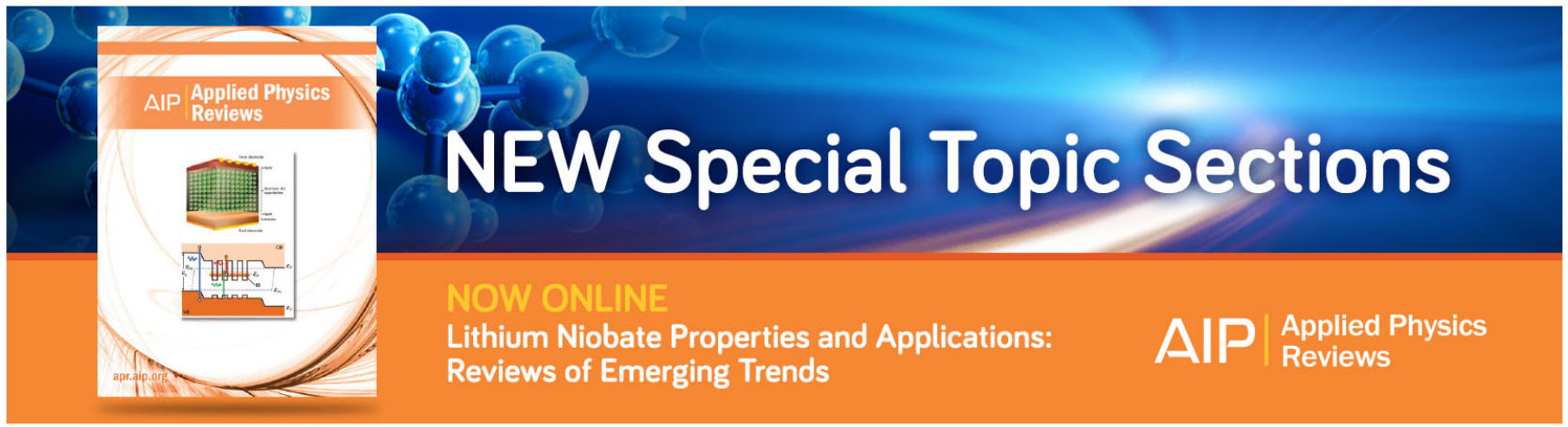




\title{
Photon management with nanostructures on concentrator solar cells
}

\author{
J. Buencuerpo, a) J. M. Llorens, M. L. Dotor, and J. M. Ripalda \\ IMM-Instituto de Microelectrónica de Madrid (CNM-CSIC), E-28760 Tres Cantos, Madrid, Spain
}

(Received 3 June 2013; accepted 5 August 2013; published online 20 August 2013)

\begin{abstract}
Optimizing the feature sizes of dielectric nanostructures on the top $(\mathrm{ZnS})$ and bottom $\left(\mathrm{SiO}_{2}\right)$ surfaces of a $1 \mu \mathrm{m}$ thick GaAs solar cell, we obtain a higher efficiency (34.4\%) than a similar cell with a state of the art bilayer antireflection coating and a planar mirror (33.2\%). The back side nanostructure increases the photocurrent due to enhanced optical path length inside the semiconductor, while the nanostructure on the front side increases the photocurrent due to lower reflectance losses. (C) 2013 AIP Publishing LLC. [http://dx.doi.org/10.1063/1.4819100]
\end{abstract}

Solar cells made of III-V compounds are the most efficient, ${ }^{1}$ but still far below the Shockley-Queiser limit. ${ }^{2}$ In the last 40 years most of the research on concentrator solar cells has been focused on increasing the quality of the semiconductor material and improving the matching of the band gaps with the solar spectrum in multi junction tandems. However, another fundamental aspect concerning the performance of a solar cell is the photon management, i.e., the optical design. Efficiency limitations related to photon management are reflection losses, the limited absorptivity of the semiconductors, and photon reemission. Rather than nanostructuring the semiconductor active layers as in previous works found in the literature, ${ }^{3-7}$ we propose to introduce dielectric nanostructures on top of concentrator solar cells, leaving the active layers intact. The results are applicable to most PV technologies, however the added cost of nano-fabrication is most advantageous in high concentration devices, where the impact of solar cell cost on the cost of the produced electricity is divided by the concentration factor.

We have used the detailed balance theory for calculating the open circuit voltage, the short circuit current, and the efficiency. ${ }^{2}$ The Sun and the solar cell are modeled as blackbody emitters. Following the notation used by Shockley, ${ }^{2}$ we have

$$
F_{\mathrm{s}}(E, \theta, T)=\iint_{0}^{\theta_{\max }} d E d \Omega a(E, \theta) b(E, T) \cos (\theta) / \pi,
$$

$F_{\mathrm{S}}$ being the total absorbed photon flux from the Sun. The incidence polar angle $\theta<\theta_{\max }$ is limited by the concentration ratio and centered around the surface normal. The blackbody spectrum $b(E, T)$ at $T=6000 \mathrm{~K}$ is used as a simplified model of solar spectrum. The solar cell is also a blackbody with thermal photon emission. The total radiative flux per unit area from the solar cell at zero bias voltage is $F_{\mathrm{c} 0}$, and is given by the same expression as $F_{\mathrm{s}}$, but with $T=300 \mathrm{~K}$ and $\theta_{\max }=\pi / 2$. For a solar cell in equilibrium or with constant quasi-fermi levels $(\mu=q V)$ within the semiconductor, the absorptance $a(E, \theta)$ is equal to the emittance $e(E, \theta){ }^{8} \mathrm{~A}$ reduced absorptance for incident angles outside the integration range of $F_{\mathrm{s}}$, i.e., $\theta>\theta_{\max }$ reduces $F_{\mathrm{c} 0}$ without affecting $F_{\mathrm{s}}$, yielding a higher open circuit voltage. The absorption of

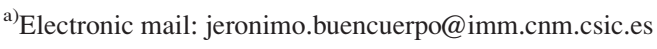

one photon with energy above the semiconductor gap is assumed to produce one hole/electron pair. ${ }^{9}$

The photocurrent density $J$ is given by

$$
J=J_{\mathrm{sc}}+q F_{\mathrm{c} 0}\left(1-\exp \left(q V / k T_{\mathrm{c}}\right)\right) .
$$

The previous equation defines a short circuit current $J_{\mathrm{sc}}=q\left(F_{\mathrm{s}}-F_{\mathrm{c} 0}\right)$, and an open circuit voltage, $V_{\mathrm{oc}}$

$$
V_{\mathrm{oc}}=\frac{k T_{\mathrm{c}}}{q} \log \left(\frac{F_{\mathrm{s}}-F_{\mathrm{c} 0}}{F_{\mathrm{c} 0}}\right) .
$$

The efficiency ( $\eta$ ) of the solar cell, with surface $A_{\mathrm{c}}$, is defined as the ratio between the maximum output power, $P_{\text {out }}$, and the incident power, $P_{\text {inc }}$

$$
\eta=P_{\text {out }} / P_{\text {inc }}=\frac{\max (J(V) V) A_{\mathrm{c}}}{P_{\text {inc }}} .
$$

Our proposal is to use dielectric periodic nanostructures and optimize their performance by calculating the resulting photocurrent, voltage, and efficiency. We have modeled a single junction GaAs solar cell with two periodic nanostructures, one made of nanostructured $\mathrm{ZnS}$ on the top of the semiconductor to act as antireflection layer, and another at the rear contact acting as a diffraction grating in order to achieve a light trapping system. The cases studied (Fig. 1) are front nanostructure only (FNO), back nanostructure only (BNO), and front and back nanostructure (FBN). These designs are more compatible with state of the art concentrator solar cell technology than previous proposals, ${ }^{4-6}$ as nanostructuring the active layer increases surface recombination and complicates current extraction. Similarly, nanostructuring the window layer inevitably increases parasitic absorption losses, as the semiconducting materials used in window layers have much higher absorption than those used in dielectric antireflective layers. ${ }^{3}$

The absorptance is calculated using the scattering matrix method, ${ }^{10,11}$ in particular we have used the ISU-TMM code with standard refractive index data. ${ }^{12}$ The efficiency was maximized using a local optimization algorithm with the nanostructure dimensions as adjustable variables. ${ }^{13}$

All modeled solar cells have a $1 \mu \mathrm{m}$ thick GaAs active layer, a lossless mirror on the back side as back contact, and a lossless concentrator with a geometrical concentration of 
(a)

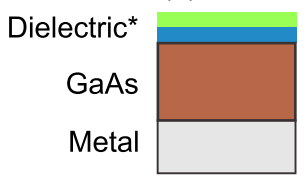

(b)

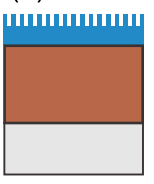

(c)

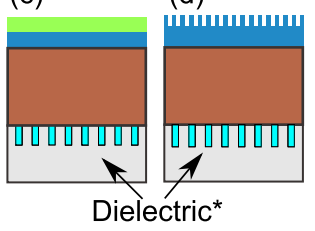

FIG. 1. (a) Bilayer antireflection coating made of $\mathrm{ZnS}$ and $\mathrm{MgF}_{2}$, (Reference System) (b) Front ZnS nanostructure only (FNO), (c) A bilayer as antireflection coating and a BNO and (d) FBN. Dielectric*: $\mathrm{MgF}_{2}$ (top green), $\mathrm{ZnS}$ (blue) or $\mathrm{SiO}_{2}$ (bottom cyan).

$X=500$ suns. The reference system (Fig. 1(a)) has an optimized $\mathrm{MgF}_{2} / \mathrm{ZnS}$ bilayer as antireflection coating (ARC) on the front side with thicknesses of $85.15 \mathrm{~nm}$ and $43.14 \mathrm{~nm}$, respectively. ${ }^{14}$ In the FNO (Fig. 1(b)) and FBN cases (Fig. 1(d)), the top nanostructure is a square lattice of $\mathrm{ZnS}$ nanopillars on top of a $\mathrm{ZnS}$ thin film with thickness $d$. For BNO (Fig. 1(c)) and FBN (Fig. 1(d)), the back nanostructure is a square lattice of $\mathrm{SiO}_{2}$ nanopillars acting as diffraction grating embedded in the metallic back contact. Each nanostructure is defined by its lattice parameter $a$, nanopillar height $h$, and the radius of the nanopillars $r$. Other nanostructures like nanoholes and different types of lattices like triangular were also considered for the FNO case. Lower efficiencies were obtained and therefore these structures were disregarded from the current study.

The feature sizes of the nanostructures in the FNO and BNO cases were optimized following 3 steps:

1. The parameters $h$ and $d$ were optimized keeping fixed values for $a$ and $r$.

2. Conversely $a$ and $r$ were optimized keeping fixed the optimal values found in step 1.

3. Finally $h, d, a$, and $r$ were optimized using the values found in steps 1 and 2 as seed.

In the BNO case, the ARC is the same as in the reference system. The feature sizes of the final nanostructures are shown in Table I. The efficiency, short circuit current density, and open circuit voltage for each system are shown in Table II.

The nanostructured devices show higher efficiencies (34.0\% vs $33.2 \%)$ and higher short-circuit currents (21.35 vs $20.83 \mathrm{~A} / \mathrm{cm}^{2}$ ) than the reference system. In the FNO case, the enhancement comes mainly from lower reflection losses, as shown in Fig. 2(a). There are reflectance oscillations at energies near the GaAs band gap in the reference and FNO case, which are Fabry-Pérot resonances. ${ }^{15}$ The cause of the lower reflectivity in the FNO case is twofold. The periodicity of the nanostructure $(a=343.70 \mathrm{~nm})$ is such that only the zeroth diffraction order is reflected back into air at normal incidence (the first diffraction order appears at $3.6 \mathrm{eV}$ ), whereas several diffraction orders are excited within the solar cell due to the higher refractive index of the

TABLE I. Summary of optimal structural parameters.

\begin{tabular}{lcccc}
\hline \hline & $h(\mathrm{~nm})$ & $d(\mathrm{~nm})$ & $a(\mathrm{~nm})$ & $r(\mathrm{~nm})$ \\
\hline FNO & 110.7 & 44.8 & 343.7 & 111.3 \\
BNO & 451.8 & - & 637.9 & 274.16 \\
\hline \hline
\end{tabular}

TABLE II. Summary of efficiencies, short circuit currents and open circuit voltage for the systems studied.

\begin{tabular}{lccc}
\hline \hline & $\eta(\%)$ & $V_{\mathrm{oc}}(\mathrm{V})$ & $J_{\mathrm{sc}}\left(\mathrm{A} / \mathrm{cm}^{2}\right)$ \\
\hline Reference & 33.2 & 1.331 & 20.836 \\
FNO & 34.0 & 1.331 & 21.349 \\
BNO & 33.8 & 1.329 & 21.219 \\
FBN & 34.4 & 1.329 & 21.631 \\
\hline \hline
\end{tabular}

semiconductor. The ratio of available diffraction orders inside vs. outside of the solar cell plays an important role in solar cell light trapping. ${ }^{7,16}$ Because of the low diffraction efficiency in the FNO case, the optical modes inside the slab are not significantly perturbed and the Fabry-Pérot resonances dominates at low energies, where the GaAs absorptivity is low. Additionally the FNO nanostructure at low energies can be described as an effective medium and behaves as a dielectric layer with an effective index. ${ }^{17}$ This refractive index can be optimized by modifying the nanostructure dimensions, i.e., the nanostructure has an additional degree of freedom compared to the bilayer, and a lower reflectivity can be achieved.

In concentration the photon flux from the Sun reaches the cell only at small incidence angles, thus increasing the absorptance at incidence angles $\theta>\theta_{\max }$ not only does not increase the generated photocurrent, but reduces the output voltage as it increases the luminescence photon flux $F_{\mathrm{c} 0}$, which is detrimental to the voltage as shown in Eq. (3). A solar cell that uses a light trapping scheme to maximize absorption, such as a Lambertian scatterer, ${ }^{18}$ and/or an antireflection coating, is likely to pay a voltage penalty due to
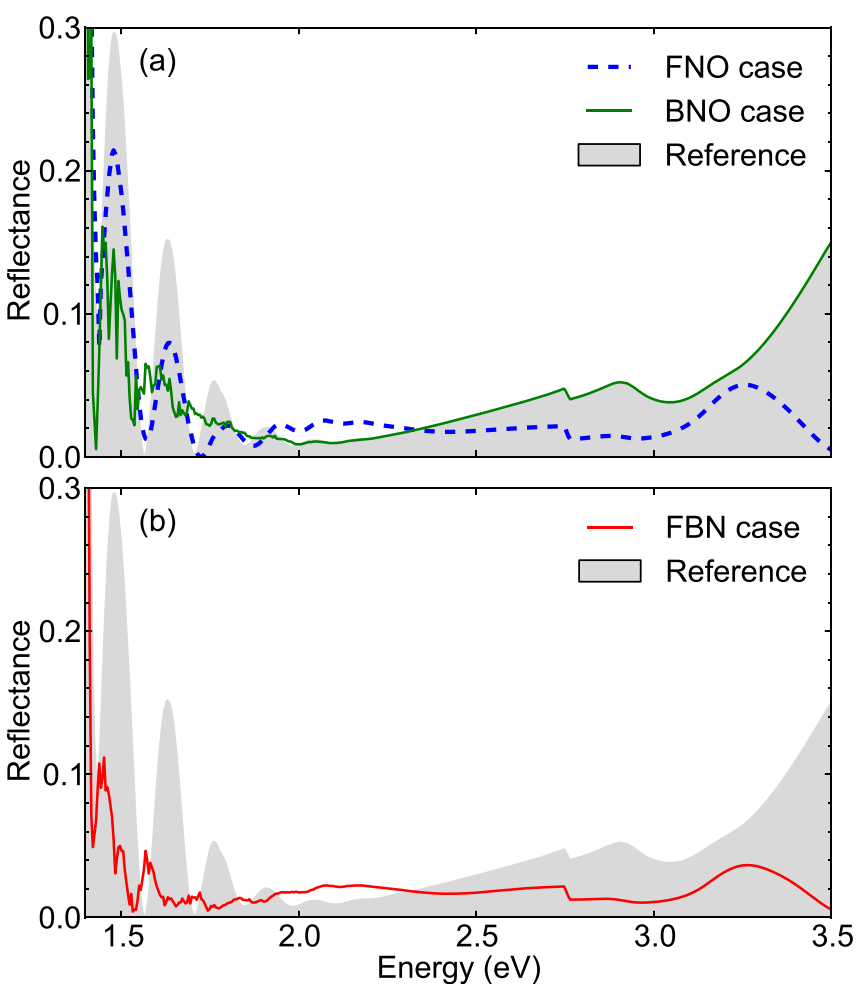

FIG. 2. Normal incidence reflectance for the reference structure (shadowed area) and (a) FNO (blue dashed line) and BNO (green line) cases, (b) FBN case (red line). 
luminescence at angles outside the incoming light cone. ${ }^{19}$ To illustrate this effect we have calculated a similar case but with an ideal ARC with zero reflection regardless of the incidence angle. Such device has $34.6 \%$ efficiency and $V_{\mathrm{oc}}=1.332 \mathrm{~V}$. Adding on top a Lambertian diffuser that isotropically scatters light increases the efficiency to $35.1 \%$ due to the increased optical path length, but reduces the $V_{\text {oc }}$ to $1.326 \mathrm{~V}$ due to the increased luminescence losses. In the case of the nanostructured systems that we have optimized there is no significant degradation of $\mathrm{V}_{\mathrm{oc}}$. To further analyze this question we present plots of the emittance as a function of energy and angle in Fig. 3. In the critical spectral region for luminescence, i.e., at energies near the band gap, the FNO is similar to the reference case; therefore, the output voltage is not significantly affected. A figure with an extended energy range is also available in the supplementary material, where the FNO case exhibits a strong angular dependence at high energies due to the closing of all diffraction channels at angles near the surface normal. Furthermore, for real GaAs devices, the non-radiative recombination current is of the same order of magnitude as radiative recombination currents, ${ }^{20}$ and as the former are unaffected by the solar cell modifications here proposed, the slight decrease in output voltage, which is a drawback of the present proposal, are of less practical importance. In practice, the impact of radiative recombination losses would only be crucial in solar cells highly optimized for light trapping, with junction widths of a few hundred $\mathrm{nm}$ at most, as non-radiative recombination is proportional to junction width.

The BNO structure is able to diffract the transmitted light and the optical Fabry-Pérot modes are perturbed as is shown in Fig. 2(a). The coupling of diffraction channels to the guided modes of the semiconductor slab is responsible for the low energy narrow peaks in Fig. 2(a), ${ }^{6,7,21}$ leading to an increase in the absorptance and consequently an increase in efficiency. For the BNO case, $J_{\mathrm{sc}}$ and $\eta$ are enhanced as the back diffraction grating increases the optical path length. A drawback of this scheme is the enhanced luminescence coupling at large emission angles away from the surface normal, as discussed before, but in this case leads to a small decrease in $V_{\text {oc }}$ compared to the reference system, see Figs. 3(c) and 3(a).

Finally, we try to exploit the benefits of the FNO and BNO devices by combining them into a single one, the FBN case. Its design starts from the optimized values of the FNO and BNO structural parameters (Table I) without any further optimization. $^{22}$ The complicated angle and energy dependence observed in Fig. 3(c) is smoothed out when the effects of both the front and back nanostructures are combined in Figs. 2(b) and 3(d). The FBN system shows an increase of $1.2 \%$ efficiency and $0.8 \mathrm{~A} / \mathrm{cm}^{2}$ in short-circuit current, see Table II. Hence, the FBN case results in a significant reduction in reflectance losses and increases the optical path length resulting in an increase of the photocurrent. The resulting voltage is the same as in the BNO case and slightly reduced in comparison to the FNO and reference cases, as expected due to the higher emittance at angles out of the incidence cone (Fig. 3(d)).

In conclusion, we have studied solar cells embedding optimized periodic nanostructures in front and back-side dielectric coating layers $\left(\mathrm{ZnS}\right.$ and $\left.\mathrm{SiO}_{2}\right)$ to increase the efficiency in concentrator solar cells without nanostructuring the active layer. Two complementary approaches were calculated: nanostructures at the top, and nanostructures at the bottom of a $1 \mu \mathrm{m}$ GaAs solar cell. The physical mechanisms enabling increased efficiencies in the present proposal can be summarized as: (1) the restriction of the open diffraction channels in air to the zeroth order while simultaneously exciting several diffraction channels inside the semiconductor, (2) the coupling of the diffraction channels to guided modes in the semiconductor slab, and (3) the index matching with the incident medium due to the effective refractive index of the nanostructure. The top nanostructure is clearly a
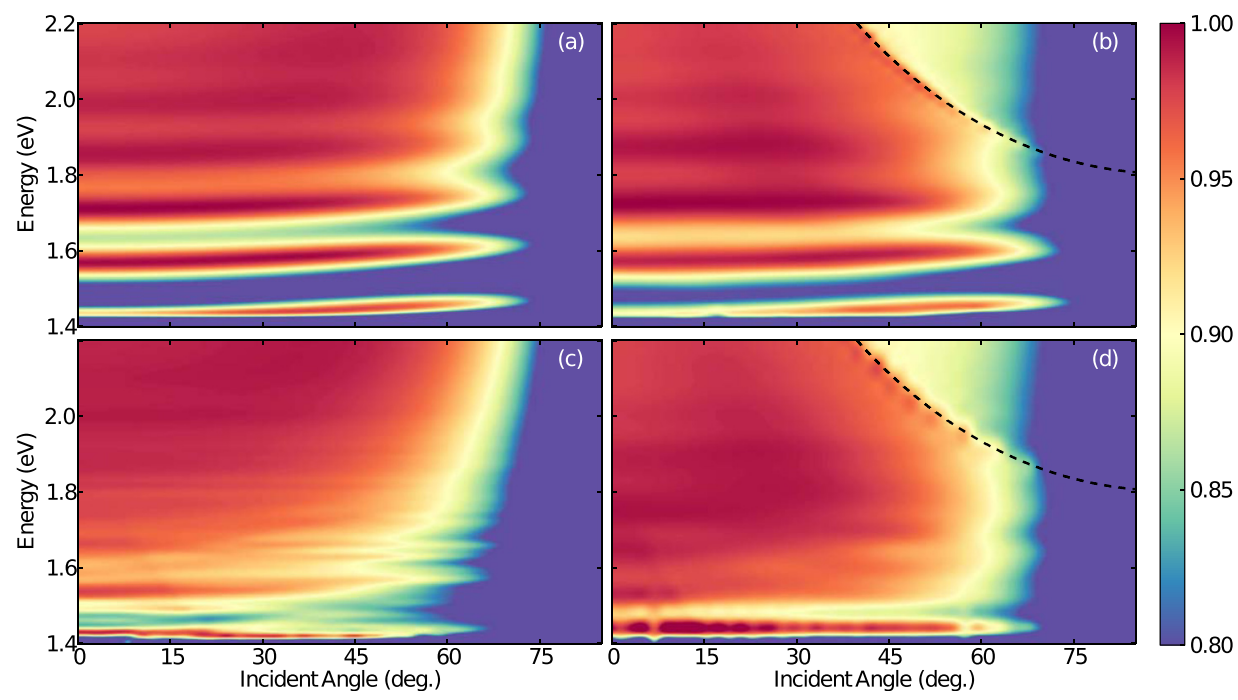

FIG. 3. Emittance as a function of the incidence angle for the Reference (a), FNO (b), BNO (c), and FBN (d) cases. The emittance is almost one for near normal incidence angles and decreases to less than 0.8 for angles larger than $75^{\circ}$. The emittance is increased for low energies near the band gap and for angles between cases 0-60 degrees in cases BNO and FBN, producing a slight loss in voltage due to radiative recombination. The dashed line indicates the threshold for the onset of higher diffraction orders in air. At near normal incidence only the zero diffraction order exists in air, while several orders exist inside the semiconductor for all angles due to the higher refractive index. The reflectance is increased as more diffraction orders open in the incident medium, and so the emittance decreases. 
better antireflection coating than standard, state of the art bilayer coatings, and there was no significant degradation in the voltage due to the inevitably higher luminescence. The bottom nanostructure increases absorption due to longer optical path lengths inside the semiconductor, and consequently increases the short circuit current. Finally, a solar cell nanostructured on both sides has been proposed showing an absolute efficiency increase of $1.2 \%$ compared to a reference cell using state of the art antireflection coatings and a perfect backside mirror.

We acknowledge the SGAI-CSIC for the allocation of computational resource and the financial support by MINECO (ENE2012-37804-C02-02, FPI grant) and CAM (S2009/ENE-1477).

${ }^{1}$ M. A. Green, K. Emery, Y. Hishikawa, W. Warta, and E. D. Dunlop, Prog. Photovoltaics 20, 12-20 (2012).

${ }^{2}$ W. Shockley and H. J. Queisser, J. Appl. Phys. 32, 510 (1961).

${ }^{3}$ I. Prieto, B. Galiana, P. A. Postigo, C. Algora, L. J. Martínez, and I. Rey-Stolle, Appl. Phys. Lett. 94, 191102 (2009).

${ }^{4}$ L. Hu and G. Chen, Nano Lett. 7, 3249 (2007).

${ }^{5}$ A. Bozzola, M. Liscidini, and L. C. Andreani, Opt. Express 20, A224 (2012).

${ }^{6}$ J. Buencuerpo, L. E. Munioz-Camuniez, M. L. Dotor, and P. A. Postigo, Opt. Express 20, A452 (2012).

${ }^{7}$ S. Zanotto, M. Liscidini, and L. C. Andreani, Opt. Express 18, 4260-4274 (2010).

${ }^{8}$ G. L. Araújo and A. Martí, Sol. Energy Mater. Sol. Cells 33, 213 (1994).
${ }^{9}$ Our calculations overestimate the photocurrent for energies above $3 \mathrm{eV}$ as our methodology cannot separate the absorption in the ARC from the absorption in the GaAs.

${ }^{10}$ M. Li, Z. Y. Li, K.-M. Ho, J. R. Cao, and M. Miyawaki, Opt. Lett. 31, 262 (2006).

${ }^{11}$ M. Li, X. Hu, Z. Ye, K.-M. Ho, J. Cao, and M. Miyawaki, Opt. Lett. 31, 3498 (2006).

${ }^{12}$ E. D. Palik, Handbook of Optical Constants of Solids II (Academic Press, New York, 1991).

${ }^{13}$ M. J. D. Powell, Acta Numerica 7, 287 (1998).

${ }^{14}$ A. Luque and S. Hegedus, Handbook of Photovoltaic Science and Engineering (Wiley, New York, 2003).

${ }^{15}$ See supplementary material at http://dx.doi.org/10.1063/1.4819100 reflectance curves for infinitely thick GaAs substrates are shown in the supplementary materials.

${ }^{16}$ Z. Yu, A. Aaswath, and S. Fan, Proc. Natl. Acad. Sci. 107, 17491-17496 (2010).

${ }^{17}$ J. E. Lugo, J. A. del Rio, and J. Tagüeña-Martínez, J. Appl. Phys. 81, 1923-1928 (1997).

${ }^{18}$ M. A. Green, Prog. Photovoltaics 10, 235-241 (2002).

${ }^{19}$ O. D. Miller, E. Yablonovitch, and S. R. Kurtz, IEEE J. Photovoltaics 2, 303 (2012).

${ }^{20}$ D. Ding, S. R. Johnson, S.-Q. Yu, S.-N. Wu, and Y.-H. Zhang, J. Appl. Phys. 110, 123104 (2011).

${ }^{21}$ R. Biswas, J. Bhattacharya, B. Lewis, N. Chakravarty, and V. Dalal, Sol. Energy Mater. Sol. Cells 94, 2337-2342 (2010).

${ }^{22}$ In order to be able to use periodic boundary conditions with a simple unit cell in the FBN case, the period of the back nanostructure was changed from the optimal value of $637.9 \mathrm{~nm}$ found in the BNO case to $687.4 \mathrm{~nm}$, i.e., twice the optimal value found in the FNO case. To minimize the impact of this change, the ratio between lattice parameter and nanopillar radius was kept the same as in the BNO case. 